\title{
EEG based Spatial Attention Shifts Detection using Time-Frequency features on Empirical Wavelet Transform
}

\section{Ampirik Dalgacık Dönüşümünde Zaman-Frekans Özniteliklerini Kullanarak EEG tabanlı Uzamsal Dikkat Kayması Tespiti}

\author{
${\text { Gokhan } \text { ALTAN }^{1} \text {, Gulcin INAT }}^{1}$ \\ ${ }^{1}$ Department of Computer Engineering, Iskenderun Technical University, Hatay, Turkey \\ ORCIDs: 0000-0001-7883-3131, 0000-0002-2923-0040 \\ E-mails: gokhan.altan@iste.edu.tr, gulcininat@gmail.com
}

\begin{abstract}
The human nervous system has over 100b nerve cells, of which the majority are located in the brain. Electrical alterations, Electroencephalogram (EEG), occur through the interaction of the nerves. EEG is utilized to evaluate event-related potentials, imaginary motor tasks, neurological disorders, spatial attention shifts, and more. In this study, We experimented with 29-channel EEG recordings from 18 healthy individuals. Each recording was decomposed using Empirical Wavelet Transform, a time-frequency domain analysis technique at the feature extraction stage. The statistical features of the modulations were calculated to feed the conventional machine learning algorithms. The proposal model achieved the best spatial attention shifts detection accuracy using the Decision Tree algorithm with a rate of $89.24 \%$.
\end{abstract}

Keywords-Electroencephalogram (EEG); empirical wavelet transform; EWT; k-NN; SVM; MLP; decision tree

$\ddot{O ̈}_{z}$ etçe-İnsan sinir sistemi, çoğunluğu beyinde bulunan 100 milyardan fazla sinir hücresine sahiptir. Elektriksel değişiklikler, Elektroensefalogram (EEG), bu sinirlerin etkileşimi yoluyla meydana gelir. EEG, olaylarla ilgili potansiyelleri, düşünsel motor görevleri, nörolojik rahatsızlıkları, mekansal dikkat kaymalarını ve daha birçok durumu değerlendirmek için kullanılır. Bu çalışmada, 18 sağlıklı bireyden alınan 29-kanallı EEG kayıtları ile analizler gerçekleştirilmiştir. Her kayıt, öznitelik çıarma aşamasında bir zaman-frekans alanı analiz tekniği olan Ámpirik Dalgacık Dönüşümü kullanılarak ayrıştırılmıştır. Sonrasında, ayrıştırılan her modülasyondan hesaplanan istatistiksel öznitelikler, geleneksel makine öğrenme algoritmalarının beslenmesinde kullanılmıştır. Önerilen model, Karar Ağacı algoritmasın kullanarak \%89.24 oranıyla en iyi uzamsal dikkat kayması algılama başarısını elde etmiştir.

Anahtar Kelimeler-Elektroensefalografi (EEG); ampirik dalgacık dönüşümü; ADA; $k-N N ; D V M$; MLP; karar ağacı

\section{INTRODUCTION}

The human nervous system is a complicated pattern with billions of nerve cells that interact. Each brain cell interacts with about 5000-50000 nerve cells. The interaction between the cells in the brain results in electrical fluctuations that invasive and non-invasive methods can measure. The electroencephalogram (EEG) is a biomedical signal type that records the electrical activity causing by brain functionality at even sleeping [1], [2]. EEG is recorded by conductive sticking the electrodes, which are not harmful and have any value of electricity or radiation to the brain. The electrodes only measure the fluctuations which present the brain activity. For this reason, It is applicable without harm to people of all ages, including pregnant women. These electrodes are commonly used to provide human-machine interaction and measure the brain response and voltage fluctuations in any external interaction [3]-[6], [8].

The amplitudes of electrical fluctuations in the brain vary in the range of $0.5-70 \mathrm{~Hz}$ within the range of $5-400 \mu \mathrm{V}$. [9]. The EEG values vary considerably depending on the recording region and EEG device specifications. Therefore, the locations of the electrodes should be predetermined to have standardization in the recordings. Although each procedure can have different channel features, brain regions used for certain sensory and motor stimuli may vary depending on the EEG device specifications. Figure 1 indicates the electrode regions and names on the skull for a 64-channel EEG device [10].

EEG recording can be performed even in case of sleep or insomnia for a complete analysis. It is possible to record EEG in many ways, such as looking at a particular picture, looking at a light at specific intervals, imagery tasks, motor tasks, and more, depending on the disease or disease findings. The test metrics are utilized as a diagnostic tool whether the patient has a neurological disorder or not. Many diseases, includ- 


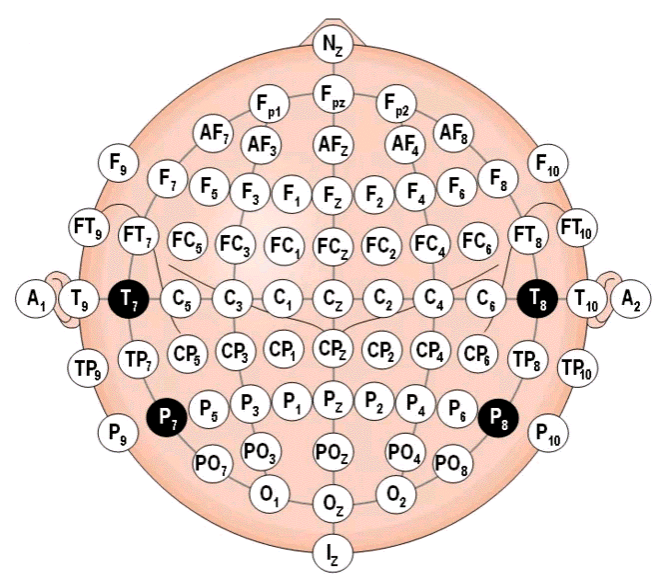

Figure 1: EEG channels commonly utilized in the literature

ing epilepsy, brain trauma, brain tumours, stroke, dementia, schizophrenia, cortical visual impairment, can be diagnosed using the EEG. Many studies focus on EEG analysis for various brain activities.

Altan et al. studied classifying brain activities from SCP training in stroke patients, used a $10 \mathrm{~Hz}$ band-pass filter, applied feature extraction with Hilbert Huang transform (HHT), and classified using DBN. They achieved the highest accuracy with a rate of $91.15 \%$ for the separation of positive and negative events. Reichert et al. analysed EEG recordings for the detection of spatial attention shifts. They also included electroretinogram (EOG) recordings to evaluate the statistical correlation. Through EEG and EOG recordings, they asked different questions with a sequence of ten visual stimuli to 18 subjects. Subjects were led to look at the green cross $(+)$ on the screen if the answer was 'YES'; otherwise, the red cross $(\mathrm{X})$ for the answer 'NO'. There are 30-channel recordings available in the database, but they experimented with 14 channels sampled at $250 \mathrm{~Hz}$. They pre-processed each recording with a $1.0 \mathrm{~Hz}$ to $12.5 \mathrm{~Hz}$ band-pass filter for artefact removal. They performed canonical correlation analysis, leaveone-out cross-validation for spatial estimation using ANOVA statistical test. They correctly estimated subject responses for visual stimuli with an average value of $88.5 \%$ (from $70.8 \%$ to 90.3\%) [11].

Honzik analysed four EEG datasets to evaluate the impact of deep learning architectures to detect various stimuli. They focused on spatial attention shifts detection as well. However, they included 14 of 29 channels in the experiments. They applied the same pre-processes in [11]. They fed the EEG plots into the various size of convolutional neural network architectures, including $5 \times 5$ filter size with 32 filters in 2 convolutional layers, $3 \times 3$ filter size with 64 filters single convolutional layer, and LTSM. He reported a compile error in iterations for the LSTM network. Moreover, deep learning architectures reached an average rate of 50\% with underperformance test results [12].

Empirical wavelet transform (EWT) is a decomposition technique that extracts adaptive wavelets over tight frames for time-series signals and images. It perfectly decomposes the input signal into different modulations by dividing the input into separate passbands by the pre-defined EWT coefficients. EWT was first developed by Gilles and Heal for using a histogram-based approach to segment the spectra [13].

Anuragi et al. studied the automatic detection of people with and without alcohol using EWT analysis on EEG signals. They also used HHT to obtain time-frequency-domain characteristics. They segmented the Fourier spectrum to overcome the boundary detection problem. They performed conventional machine learning algorithms with leave-one-out technique to classify time-frequency features on support vector machine (SVM), and k-Nearest Neighbour (k-NN). They achieved the highest performance with a success rate of $98.75 \%$ using least square-based SVM [14].

Bhattacharyya et al. utilized EWT for automatized detection of the focus and out-of-focus on EEG signals. They also separated the EEG signal into rhythm sections. The LS-SVM algorithm achieved binary classification accuracy rates of $90 \%$ and $82.53 \%$ for 50 and 750 pairs of signals, respectively [15].

Demir et al. analysed auscultation sounds using EWT for faster classification of a common pulmonary disease. They worked on a database consisting of multi-channel lung sounds by extracting statistical features from the modulations using nine machine learning algorithms. AdaBoost algorithm reached the most successful classification results with rates of $90.00 \%$, $100.00 \%$, and $95.00 \%$ for sensitivity, specificity, and overall accuracy [16].

Sadiq et al. used the EWT-based signal decomposition method on the motor imagery EEG database to improve the classification performance. They analysed 128-channel EEG signals from 5 healthy participants sitting in a chair. 118 EEG channels were recorded at positions according to the expanded international 10/20 system. They selected 18 motor cortex channels rather than using all channels by separating each channel into ten adaptive frequency modes. Hilbert Transform was applied to each decomposed mode. High classification performances were reported in most of the folds with the rates of $92.55 \%$. In contrast, the average classification performance rates for the IF1 experiment across all floors were $94.44 \%$, $86.11 \%, 97.22 \%, 100 \%$, and $72.22 \%$ for the 5 participants [17].

Akbari and Ghofrani studied EEG signals for evaluating focus and localization capabilities on epilepsy. They decomposed EEG signals for localization of different areas with drugresistant epilepsy patients before brain surgery to identify the focus and non-focus specifications. It is time-consuming with human error for detection of focus on EEG. Therefore, in their study, Akbari et al. proposed an automatic and adaptive method based on the second-order difference plot (SODP) and EWT modulations on EEG as an adaptive signal separation. They selected 50 pairs of EEG signals with a sampling frequency of $512 \mathrm{~Hz}$ from 5 epilepsy patients candidates for a brain operation to evaluate the focus and non-focus groups. They fed k-NN and SVM classifiers with 10-fold cross-validation using EWT mode functions and SODP features to provide data variability rates. They reached accuracy rates of $91.00 \%$ and 93.00\% with the SVM and k-NN, respectively [18].

Researchers applied various transformation techniques on 
the EEG signal to determine specific mental and neurological information. Most of them directly start the pattern recognition process using statistical analysis on long-term raw EEG signals. However, it is more appropriate to decompose different time-frequency domains and following feature extractions to obtain deterministic signal modulations. Since the EEG data is a signal that is prone to noise, analysing it in both frequency and time domains will enable the problem to be handled with more deterministic features. In this study, in light of these necessities, we utilized pre-processing to the EEG to noise removal, frequency-time domain analysis with the EWT, and classification with conventional machine learning algorithms. The remaining paper is organized as sequential applications of data preparation, including segmentation and pre-processing, EWT in detail, experimental setup for classification algorithms, and achievements with a complete comparison to state-of-theart.

\section{MATERIAL AND Methods}

\section{A. Database}

EEG is a frequently referenced biomedical signal for braincomputer interfaces. We evaluated open access Brain/Neural Computer Interaction: Horizon 2020 EEG database to identify spatial attention shifts to coloured items [19]. The EEG database is comprised of 18 subjects that were presented with a sequence of ten visual stimuli. Each subject has a 29channel EEG and EOG recordings from healthy participants. Each recording contains three structures: subject information including age, gender, hand preference, the native language; Eyemov that contains all recordings of both EEG and EOG data from the first recording of eye movements from the EOG stimuli; Bciexp that includes EEG signals, vertical and horizontal stimuli metrics for EOG.

During the EEG augmentation procedure, 18 healthy subjects were asked to look at the green "+"-cross and the red " $\mathrm{x}$ "-cross without any questions in the first two trials. In trials 3-6, only objective questions were asked to look “+"'-cross for yes respond and " $x$ "-cross for no response. In the remaining trials, subjective questions were asked to the subjects with the same visual stimuli. There are 24 trials in total.

EEGs were recorded with a Brainamp DC amplifier using 30-channel $\mathrm{Ag} / \mathrm{AgCl}$ electrodes referenced against the right mastoid as well as horizontal and vertical EOG. It has a sampling rate of $250 \mathrm{~Hz}$ and a high-pass filter with $0.1 \mathrm{~Hz}$ to reduce the noise. The 29th channel of the recording device is set as the reference channel. The channel names in order: Fp1, Fp2, F7, F3, Fz, F4, F8, FC1, FC2, T7, C3, Cz, C4, T8, O9, CP1, CP2, O10, P7, P3, Pz, P4, P8, PO7, PO3, Oz, PO4, PO8, LMAST, and IZ [11]. In this study, the experiments were carried out on the first channel of subjects for various classification algorithms.

\section{B. Empirical Wavelet Transform}

Empirical wavelet transform (EWT) is a time-frequency analysis technique to decompose spatial information from different signal types. The EWT detects the local maxima of the Fourier spectrum of the signal in the first stage, then segments the modulations from EEG. The spectrum information is based on the detected maximum and finally generates a corresponding wavelet filter bank. The EWT method has sequential operations as follows [20]:

1) Specification of frequency components by fast Fourier transform

2) Extracting various modulations of the signal by attaining the actual segmentation of the Fourier spectrum

3) Performing a scaling and empirical wavelet kernels concerning each extracted segment. Segmenting Fourier spectrum from the signal is the most significant procedure in the adaption of EWT.

Empirical wavelet kernels $(\Psi(w))$, that are conducted as band-pass filters, and the empirical scaling function $\phi(w)$ are defined as follows [13]:

$$
\Psi(w)= \begin{cases}1 & (1+\gamma) \Omega_{n} \leq|w| \leq(1-\gamma) \Omega_{n+1} \\ \cos \left[\frac{\pi}{2} \beta\left(\gamma, \Omega_{n+1}\right)\right] & (1-\gamma) \Omega_{n+1} \leq|w| \leq(1+\gamma) \Omega_{n+1} \\ \sin \left[\frac{\pi}{2} \beta\left(\gamma, \Omega_{n}\right)\right] & (1-\gamma) \Omega_{n} \leq|w| \leq(1+\gamma) \Omega_{n} \\ 0 & \text { otherwise }\end{cases}
$$

and

$$
\phi(w)= \begin{cases}1 & |w| \leq(1-\gamma) \Omega_{n} \\ \cos \left[\frac{\pi}{2} \beta\left(\gamma, \Omega_{n}\right)\right] & (1-\gamma) \Omega_{n} \leq|w| \leq(1+\gamma) \Omega_{n} \\ 0 & \text { otherwise }\end{cases}
$$

where $\gamma$ is a parameter to ensure no overlap for segmented areas. Many functions commonly used in the literature meet these specifications. Inner modulations obtain detailed coefficients. Empirical scaling function is an FFT representation of EEG recording. The approximation coefficients are extracted using inner modulations (detailed in [21]).

\section{Classification}

Classification algorithms are frequently used to categorize the feature vectors with an output function corresponding to the similarity functions and weighted values. In this study, we evaluated the identification performances for spatial attention shifts to coloured items using conventional machine learning algorithms, including k-NN, decision tree, SVM, and multilayer perceptron (MLP).

The k-NN algorithm is one of the simplistic distancebased machine learning procedure. The learning is carried out concerning the training set. A newly encountered sample is classified concerning the distance-based similarity between the distributed samples in the training [3]-[7], [22]. As a result of measuring the closeness of the samples on the dataset with Euclidean, Minkowski, Manhattan or another distance formula, it categorizes new samples into a defined class among the $\mathrm{k}$ number of class(es).

The decision tree algorithm is a graph-based classifier which consists of nodes, branches, and leaves. The nodes specify the test. Branching separation is performed as a sequential procedure using entropy-based information gain function for 
each node. Branch separation depends on distinctions that are on a high level. If a separation procedure cannot be ended at the last branch, the decision node is defined as a resulting branch in the tree. Decision tree separation procedure starts with a root node and continues defining the successive nodes in a sequential manner down to reaching the leaf node [23].

The SVM was a mostly used conventional machine learning algorithm for classification and regression problems in EEG analysis. It defines the optimum vectors to separate the training samples on a plane. It aims at maximizing the separation vector for both classes using the region between \pm 1 of margin. The wider the margin has the best generalization capability for the separation of binary and multi-class [9].

The MLP algorithm is a popular algorithm with Delta Learning Rule and adaptability of various optimization techniques. The depth of MLP depends on the number of the hidden layer(s) and nodes. The input layer receives the incoming features and transfers them to the following layer using a forward-pass procedure with linear algebra and activation functions. The number of hidden layers varies according to the problem, at least one, and is adjusted according to the depth of the analysis. The output of each neuron has a fully connection between the next layer as an input value. Each neuron has a fully-connection among the nodes at adjacent layers. The output layer predicts the output value(s) for the MLP model to converge the actual outputs using a gradient descent algorithm with a learning rate and epoch [8], [17], [22].

\section{EXPERIMENTAL RESULTS}

A pattern recognition process on EEG is comprised of data pre-processing, applying decomposition methods to get timefrequency domain modulations, feature extraction from various modulations, feature set normalisation, and classification. Due to the analysed EEG database is at online available, we started to manipulate the dataset for separating the trials. We experimented on one random subject among database to evaluate a subject-based performance for the proposed model. We segmented interval length of 0.75 for per second due to the procedure of visual stimuli presentation in EEG augmentation in [11]. This procedure provides to avoid any conflict with the database and a complete comparison with the related paper. We applied a band-pass of $1 \mathrm{~Hz}$ to $12.5 \mathrm{~Hz}$ to allowed the signal pass through the filter. The reference EEG-channel is set as 'LMAST'.

The EWT was applied to the related EEG database for the first time to decompose frequency modulations on spatial attention shifts to colored items. Hence, the proposal is a pioneering technique on the EEG analysis for detection of spatial attention shifts on 29-channel EEGs. Moreover, EWT provides a noise elimination on the raw EEG. After decomposing the modulations, we calculated A total number of 31 statistical features including skewness, mode, median, min, max, kurtosis, moments, and more for each mode using python scripts. While using this procedure, EWT was applied separately to each trial for pre-defined channel. As a result, the EWT modulations in the signal and the boundaries of the signal for the EWT are carried out for many variations.
After attaining the feature set, the normalized features were converted to CSV format to provide a standardized analysis dataset. The dataset was analysed on the JAVA-based WEKA platform using 4 conventional classification algorithms. Each classifier has unique variables and iterations. Each classification parameter has been iterated many times in different combinations. The highest identification performances for spatial attention shifts to colored items are presented in Table I for each classifier.

\begin{tabular}{|l|l|l|l|l|}
\cline { 2 - 5 } \multicolumn{1}{c|}{} & Decision Tree & k-NN & MLP & SVM \\
\hline Data Separation & 15 -fold CV & $\begin{array}{l}\% 90 \text { train } \\
\% 10 \text { test }\end{array}$ & $\begin{array}{l}\% 90 \text { train } \\
\% 10 \text { test }\end{array}$ & 10 -fold CV \\
\hline Correct / Total & $7392 / 8283$ & $573 / 828$ & $537 / 828$ & $5212 / 8283$ \\
\hline Accuracy (\%) & 89.2430 & 70.1021 & 64.8551 & 62.9241 \\
\hline MSE & 0.1348 & 0.2980 & 0.4347 & 0.3708 \\
\hline
\end{tabular}

Table I: Experimental setup and achievements for each machine learning algorithms

Since the decision tree algorithm separates the features according to statistical entropy-based difference without iteration parameters. However, validation methods and node specifications were changed in the experiments to obtain a robust comparison in terms of achievements. The dataset is divided into training and testing folds with 2 different methods: $\mathrm{k}$-fold cross-validation method $(\mathrm{k}=5,10$, and 15) and traintest folds (90\% train-10\% test, $75 \%$ train-25\% test). It has been observed that there is not much difference between the results for the decision tree algorithm in the iterations. On the other hand, whereas experimenting with the k-NN algorithm, $\mathrm{k}$ parameters $(\mathrm{k}=1,3,5,7$, and 9) were carried out for various cross-validation parameters. We also utilized the same $\mathrm{k}$-fold cross-validation parameters $(\mathrm{k}=5,10$, and 15) and dividing the dataset into train and test data (90\% training$10 \%$ test, $75 \%$ train- $25 \%$ test). The highest classification performance was achieved for 1-NN, whereas the performance gradually decreased as the $\mathrm{k}$ parameter was increased. It was observed that this decrease did not change for various validation techniques. MLP has many parameters need to be iterated and optimized for every evaluation due to the randomly definition of the starting weights between nodes. Trainings were carried out at the range of 50-250, 100-500, and 1-3 for number of neurons, epoch, and hidden layers, respectively. Due to overcome the limitations on local minimum in the training with high randomness, each MLP model was trained 3 times. The best achievements for the further trainings were presented in this study. Since there are not many parameters to change in the SVM classification algorithm, training attempts were performed on various validation techniques and division percentages on the dataset.

As it is presented in Table I, the decision tree is the best classification algorithm in the identification of spatial attention shifts to colored items among the entire experiments on machine learning algorithms and validation techniques in this study. The dataset was divided into training and testing folds (total number of 8283 EEG) using the 15 -fold cross-validation method. The proposed model with the highest classification performance correctly classified a total number of 7392 . The 
decision tree reported the minimum error value of 0.1348 and the highest test accuracy rate of $89.243 \%$ for spatial attention shifts analysis on EEG.

The k-NN classifier reached the second highest test identification performance for spatial attention shifts to colored items with an accuracy of $70.1021 \%$ for separated train and test folds. The achievements were reported with an error value is 0.298 using $90 \%$ train folds and remaining for test on 1-NN.

Various MLP models with many neurons on 1, 2, and 3 hidden layers were iterated for the dataset. The highest test identification performance for spatial attention shifts to colored items is obtained for $90 \%$ train fold and $10 \%$ test fold using 3 hidden layers (100-100-100 neurons in each layer), 10 iterations, the learning rate of 0.01 , the momentum value of 0.9 , and 500 epochs.

The highest classification performance among the SVM iterations is obtained with an accuracy rate of $62.9241 \%$ using 10 -fold cross-validation. However, in general, the achievement is not quite enough to identify spatial attention shifts to colored items on EEG with the statistical features from the EWT modulations. It is possible to get better identification results by training various non-linear kernels and more channels in detail.

\section{Discussion}

Various EEG analysis techniques are focused on directly classifying motor imagery stimuli, diagnosing neurological disorders, and activities on human-computer interaction using advantages of novel classifiers and transfer learning. In this study, we applied many different classification parameters and value ranges for conventional machine learning algorithms on time-frequency domain characteristics for identifying spatial attention shifts. We reported the highest classification performances stratifying by classifiers among the entire experimental results.

Since the analysed EEG dataset is a new database, there are a limited number of studies on the spatial attention shifts to colored items. Due to the existing literature evaluated a statistical correlation on directly raw EEG, the proposed method is a premise technique on the frequency-time domain based classification on the EEG database. Hence, a complete comparison is not possible to evaluate the performance of the machine learning algorithms. However, the achievements indicate that the proposed architecture reached high enough classification performances even for simplistic mathematical backgrounds such as the decision tree algorithm.

Reichert et al. did not use a machine learning algorithm in the analysis with directly raw EEG recordings including 10 trials and 29-channels. They reached statistically high correlation values for the given specific problem results with significance value $(p)$ of $0.298 ; \mu=89.4 \%, \sigma=8.9 \%$ and $\mu=87.5 \%, \sigma=8.1 \%$ for "yes" and "no", respectively. Their statistical-based proposal with correlation analysis was concluded that the decoder was not biased for EEG evaluation on the identification of spatial attention shifts to colored items [11].

Honzic et al. utilized machine learning algorithms on the identification of spatial attention shifts to colored items. However, their proposal did not achieve high enough classification performances for repeated experiments with the artificial neural networks and deep learning models. Although they applied efficient algorithms including convolutional neural networks and long short-term memory (LSTM) on the plotted EEG recordings with the same pre-processing procedure for each trial, the experimented architectures reported classification performances prone to almost overfitting with rates of $50.39 \%$ and $50.67 \%$, respectively [12].

\begin{tabular}{|l|l|l|c|}
\hline Related works & Methods & Classifiers & Accuracy(\%) \\
\hline Reicher et al. & - & CCA, LOOCV, ANOVA & 88.50 \\
\hline \multirow{3}{*}{ Honzik et al. } & \multirow{3}{*}{ This study } & CNN (ANN) & 50.60 \\
\cline { 3 - 4 } & \multirow{3}{*}{ EWT } & CNN (SNN) & 50.39 \\
\cline { 3 - 4 } & LTSM (ANN) & 50.67 \\
\hline & Decision Tree & 89.24 \\
\cline { 3 - 4 } & k-NN & 70.10 \\
\cline { 3 - 4 } & MLP & 64.86 \\
\cline { 3 - 4 } & SVM & 62.92 \\
\hline
\end{tabular}

Table II: Comparison of the experimental results with the stateof-the-art

Table II presents the related works for the EEG analysis on separation of "yes" and "no" responses for spatial attention shifts to colored items. Various alternatives for feature extraction methods, validation techniques on splitting train and test data, classifiers and statistical significance, and the achievements for efficient procedures are existing on the EEG analysis. The related works, which have focused on the experimented EEG recordings, reached average performances on trials and different stimulus. However, applying EWT and calculating the statistical features from each EWT modulation and feeding the conventional machine learning algorithms outperformed the state-of-the-art. The highest spatial attention shifts identification performance were achieved using subject-specific EEG recordings using Decision tree algorithm. Although the k-NN, MLP, and SVM classifiers had more inefficacious separation performances, the proposals on these classifiers reached higher performances than the state-of-the-art for even a limited range of iteration parameters.

\section{CONClusion}

Using fiducial feature extraction algorithm EWT on EEG recordings with various trials has achieved better generalization capability for even subject-specific solutions. Especially, adapting machine learning algorithms into the EEG evaluation progresses reaches a better separation performance than using statistical analysis on raw EEG. Moreover, it provides minimization of overall model error for time-frequency characteristics of the EEG recording instead of time-domain specifications. Whereas the-state-of-the-art had the deficiency of the detailed analysis on the plotted EEG, extracting EWT modulations enables significant improvements for spatial attention shifts to colored stimuli. However, the decrease in classification performance for Deep learning algorithms draws attention for the importance of transform progress and oppositely for using raw EEG. In the feature works, it is possible to perform detailed analysis on the time-frequency distributions of EWT modulation using deep learning architectures and integration of 
various frequency analysis techniques on the trial-based EEG recordings.

\section{AUTHOR CONTRIBUTIONS}

This paper is a part of Gulcin INAT's Ms thesis. Dr. Gokhan ALTAN is the thesis advisor. All authors equally contributed on analyzing EEGs and writing the paper.

\section{REFERENCES}

[1] Sayilgan E, Yuce YK, Isler Y. Determining gaze information from steadystate visually-evoked potentials. Karaelmas Science and Engineering Journal 2020; 10(2): 151-157.

[2] Altan G, Yayik A, Kutlu Y. Deep learning with ConvNet predicts imagery tasks through EEG. Neural Processing Letters 2021; 1-16.

[3] Sayilgan E, Yuce YK, Isler Y. Frequency recognition from temporal and frequency depth of the brain-computer interface based on steady-state visual evoked potentials. Journal of Intelligent Systems with Applications 2021; 4(1): 68-73.

[4] Sayilgan E, Yuce YK, Isler Y. Evaluation of mother wavelets on steadystate visually-evoked potentials for triple-command brain-computer interfaces. Turkish Journal of Electrical Engineering \& Computer Sciences 2021; 29(5): 2263-2279.

[5] Sayilgan E, Yuce YK, Isler Y. Evaluating steady-state visually evoked potentials-based brain-computer interface system using wavelet features and various machine learning methods. Book chapter in Brain-Computer Interface, IntechOpen, 2021.

[6] Sayilgan E, Yuce YK, Isler Y. Evaluation of wavelet features selected via statistical evidence from steady-state visually-evoked potentials to predict the stimulating frequency. Journal of the Faculty of Engineering and Architecture of Gazi University 2021; 36(2): 593-605.

[7] Koklu M, Sabanci K. Estimation of credit card customers payment status by using KNN and MLP. International Journal of Intelligent Systems and Applications in Engineering 2016; SI: 249-251.

[8] Altan G, Kutlu Y, Allahverdi N. Deep belief networks based brain activity classification using EEG from slow cortical potentials in stroke. International Journal of Applied Mathematics Electronics and Computers 2016; SI-1: 205-210

[9] Kantar T, Erdamar A. Detection of K-complexes in sleep EEG with support vector machines. In 25th Signal Processing and Communications Applications Conference (SIU), May 15-18, 2017, Antalya, Turkey, pp. $1-4$.

[10] Tang BB, Wei X, Guo G, Yu F, Ji M, Lang H, Liu J. The effect of odor exposure time on olfactory cognitive processing: An ERP study. Journal of Integrative Neuroscience 2019; 18(1): 87-93.

[11] Reichert C, Tellez Ceja IF, Sweeney-Reed CM, Heinze HJ, Hinrichs H, Durschmid S. Impact of stimulus features on the performance of a gazeindependent brain-computer interface based on covert spatial attention shifts. Frontiers in Neuroscience 2020; 14:1250.

[12] Pavel S. Use of spiking neural networks. BSc Thesis, University of West Bohemia, Czech Republic, 2021. Retrieved from https://dspace5.zcu.cz/handle/11025/44220

[13] Gilles J. Empirical wavelet transform. IEEE Transactions on Signal Processing 2013; 61(16): 3999-4010.

[14] Anuragi A, Sisodia DS. Empirical wavelet transform based automated alcoholism detecting using EEG signal features. Biomedical Signal Processing and Control 2020; 57: 101777.

[15] Bhattacharyya A, Sharma M, Pachori RB, Sircar P, Acharya UR. A novel approach for automated detection of focal EEG signals using empirical wavelet transform. Neural Computing and Applications 2018; 29: 47-57.

[16] Demir E. Diagnosis of chronic obstructive pulmonary disease using empirical wavelet transform analysis from auscultation sounds. MSc Thesis, Iskenderun Technical University, Hatay, Turkey, 2020.
[17] Sadiq MT, Yu X, Yuan Z, Fan Z, Rehman AU, Li G, Xiao G. Motor imagery EEG signals classification based on mode amplitude and frequency components using empirical wavelet transform. IEEE Access 2019; 7: 127678-127692.

[18] Akbari H, Ghofrani S. Fast and accurate classification F and NF EEG by using SODP and EWT. International Journal of Image, Graphics and Signal Processing (IJIGSP) 2019; 11(1): 29-35.

[19] Reichert R, Tellez Ceja IF, Durschmid S. Spatial attention shifts to colored items-An EEG-based brain-computer interface. Repository for Research Data and Publications of OVGU, 2020. Retrived from doi: 10.24352/UB.OVGU-2020-155

[20] Gursoy MI, Ustun SV, Yilmaz AS. An efficient DWT and EWT feature extraction methods for classification of real data PQ disturbances. International Journal of Engineering Research and Development 2018; 10(1): 158-171.

[21] $\mathrm{Hu} \mathrm{Y,} \mathrm{Li} \mathrm{F,} \mathrm{Li} \mathrm{H,} \mathrm{Liu} \mathrm{C.} \mathrm{An} \mathrm{enhanced} \mathrm{empirical} \mathrm{wavelet} \mathrm{transform}$ for noisy and non-stationary signal processing. Digital Signal Processing 2017; 60: 220-229.

[22] Mitchell TM. Does machine learning really work? AI Magazine 1997; 18(3): 11-20.

[23] Ozekes S. A Data mining application. PhD Thesis, Marmara University, Istanbul, Turkey, 2002 